\title{
Research Trends and Regulation of CCL5 in Prostate Cancer
}

This article was published in the following Dove Press journal: OncoTargets and Therapy

\author{
Renlun Huang ${ }^{1} * *$ \\ Lang Guo',* \\ Menghan $\mathrm{Gao} \mathbb{D D}^{2}$ \\ Jing $\mathrm{Li}^{\prime}$ \\ Songtao Xiang' \\ 'Department of Urology, The Second \\ Affiliated Hospital of Guangzhou \\ University of Chinese Medicine, \\ Guangzhou, Guangdong, People's \\ Republic of China; ${ }^{2}$ Department of Cell \\ and Molecular Biology, Uppsala \\ University, Uppsala, Sweden \\ *These authors contributed equally to \\ this work
}

\begin{abstract}
Prostate cancer ( $\mathrm{PCa})$ is considered as the most common cancer of urologic neoplasms, and its development and prognosis are associated with many factors. Chemokine receptor signaling combine with advances in advanced clinicopathological characteristics have provided new insights into the molecular landscape of prostate cancer. Chemokine (C-C motif) ligand 5 (CCL5) is an important member of the CC subfamily of chemokines. The expression of chemokine CCL5 is positively correlated with poor prognostic features in patients with PCa. Current study suggested that CCL5/CCR5 axis plays a significant role in the proliferation, metastasis, angiogenesis, drug resistance of prostate cancer cells and promotes self-renewal of prostate cancer stem cells (PCSCs). Due to the major domination in CCL5 by prostate cancer and the high cancer-specific mortality with prostate cancer, research on the CCL5/CCR5 axis effective antagonists is widespread application. However, challenges for precision oncology of CCL5/CCR5 axis and effective antagonists in CRPC remain. Herein, we summarized the crucial role of CCL5 in promoting the development of $\mathrm{PCa}$ and discussed the antitumor application of the antagonists of CCL5/CCR5 axis.
\end{abstract}

Keywords: chemokine, tumor development, CCR5, antagonists

\section{Introduction}

Prostate cancer (PCa), a kind of heterogeneous malignancy, is the most common cancer and the second most common cause of cancer-related mortality among men in the United States, with 174,650 new cases and 31,620 deaths estimated in 2019. ${ }^{1}$ Regulated by a huge variety of intrinsic and microenvironmental factors, tumor development and malignancy are multifactorial result. ${ }^{2,3}$ The microenvironmental mediator has become a promising target for PCa treatment. ${ }^{4}$ Notably, the chemokines, as microenvironmental mediators, are an important messenger mediating the development of PCa. ${ }^{4}$ For example, CCL5, the abundant chemokine derived from tumor-associated macrophages, can promote PCa metastasis. ${ }^{5}$ Increased levels of CCL5 in tissues or blood are positively associated with the poor prognosis and advanced clinicopathological characteristics of PCa. ${ }^{5}$ Accumulating evidences suggest that targeting the CCL5/CCR5 axis can be viewed as an effective antitumor strategy for PCa treatment. ${ }^{5}$ At present, the antagonists of this axis associated with $\mathrm{PCa}$ are cenicriviroc, maraviroc, anibamine and DT-13. These antagonists can exert valid antitumor effects on PCa.

Herein, we review the crucial role of CCL5 in promoting the development of $\mathrm{PCa}$ and currently available antagonists that target the CCL5/CCR5 axis for PCa treatment.
Correspondence: Songtao Xiang
Department of Urology, The Second

Affiliated Hospital of Guangzhou

University of Chinese Medicine,

Guangzhou, People's Republic of China

Tel/Fax +86-I37II 5 I 5862

Email tonyxst@gzucm.edu.cn
OncoTargets and Therapy 202I:14 1417-1427

1417 in $\square$ 


\section{Chemokines and CCL5}

Chemokines belong to the superfamily of chemotactic cytokines, ${ }^{6}$ soluble small molecule $(8-14 \mathrm{kDa})$ signaling proteins $^{7}$ whose receptors are typically seventransmembrane guanine nucleotide-binding protein (G-protein) coupled receptors (GPCRs). ${ }^{4}$ The specific binding of chemokines to chemokine receptors will activate the downstream pathway of phosphatidylinositol 3 kinase and small Rho guanosine triphosphatase. ${ }^{8}$ The first chemokine, platelet factor 4 (PF-4/CXCL4), became known in 1977. ${ }^{9}$ Since then, chemokines have been identified and divided into four subfamilies: CXC, CC, CX3C, and $\mathrm{C}$ chemokines. ${ }^{10}$ Currently, CXCLn, CCLn, CLn and $\mathrm{CX} 3 \mathrm{CLn}$ ( $\mathrm{L}$ is a ligand, $\mathrm{n}$ is a different number) are used to represent different members of the $\mathrm{CXC}, \mathrm{CC}, \mathrm{C}$, and CX3C families. ${ }^{11}$ The CC chemokines are known by the two adjacent cysteines at the amino terminus and are closely related to inflammation and the immune response. ${ }^{12}$ Recent studies have indicated that CCL22 ${ }^{13}$ and CCL5 $5^{14,15}$ can promote PCa cell migration. CCL5 was originally discovered in 1988 by analyzing cDNA libraries of subtracted T cells, ${ }^{16}$ but detailed reports on the relationship between CCL5 and PCa were not available until 2004. ${ }^{17}$ As a member of the CC subfamily, CCL5 is expressed in $\mathrm{T}$ lymphocytes, macrophages, synovial fibroblasts, tubular epithelium, endothelial cells, and selected tumor cells, including PCa cells. ${ }^{5,18}$ It mainly interacts with CCR5 while it can also bind other classic receptors CCR1, CCR3, CCR4 and GPR75 and the atypical receptors ACKR1 and ACKR2 in various cell types. ${ }^{19}$ When CCL5 recognizes and binds its receptors, such as CCR5, heterotrimeric $\mathrm{G}$ proteins and JAK family tyrosine kinases are activated to trigger multiple downstream signaling cascades thus conferring not only the proliferation, metastasis, angiogenesis, and drug resistance of prostate cancer cells, but also the self-renewal of prostate cancer stem cells (PCSCs). ${ }^{5}$

\section{The Role of CCL5 in the Development of Prostate Cancer}

Along with unceasingly thorough research, the occurrence of tumors has been realized to be related to many factors. Chemokines have a wide range of biological functions and are worthy of further study. Among them, CCL5 is the most prominent, for it is not only involved in an inflammatory reaction ${ }^{20}$ but also closely related to tumorigenesis, tumor growth, invasion and metastasis. ${ }^{2,17}$ We reviewed existing studies of CCL5 promoting the proliferation, angiogenesis, metastasis, drug resistance of prostate cancer cells and the self-renewal of PCSCs, and which are shown in Figure 1.

Dr. Vaday reported that CCL5 alone could promote the proliferation of PCa cells. ${ }^{21}$ At the same time, the combination with the CCR5 antagonist TAK-779 inhibited the proliferation of PCa cells, suggesting that CCL5 may act by binding to CCR5.$^{21}$ There is a research demonstrated that the interaction of the cell surface prostate-specific membrane antigen (PSMA) with specific antibodies strongly induce $\mathrm{NF}-\kappa \mathrm{B}$ activation and then promote the gene expression of IL-6 and CCL5. ${ }^{22}$ And CCL5/CCR5 interactions lead to the upregulation of cyclin D1 and ultimately promote the proliferation of $\mathrm{LNCaP}$ cells by promoting phosphorylation of STAT5. ${ }^{22}$ After the addition of CCR5 antibody, cell proliferation was inhibited. ${ }^{22}$ In addition, CCL 5 could also stimulate cell proliferation by activating the mTOR pathway, resulting in rapid upregulation of cyclin D1 and c-Myc. ${ }^{23}$ The mutual effect between CCL5 and CCR5 could also increase the uptake of glucose, increase ATP production and augment glycolysis in tumor cells, thereby promoting tumor cell proliferation. ${ }^{23}$

By injecting polyester-polyurethane sponges into mice to induce inflammation, researchers shown that neovascularization was associated with dynamic changes in the chemokine CCL5. ${ }^{24}$ Furthermore, exogenously added CCL5 in CCR5 knockdown C57BL/6J mice did not promote corneal neovascularization, suggesting that CCL5/ CCR5 could promote corneal neovascularization. ${ }^{25}$ In a study of ovarian cancer, the researcher identified that CCL5 promoted tumor angiogenesis by activating NF- $\mathrm{KB}$ and STAT3 pathways. ${ }^{26}$ After genetic analysis of BPH (Benign Prostate Hyperplasia) cells with CCL5 intervention, the researcher discovered that the expression of angiogenin and pleiotrophin significantly increased, both of which were potent inducers of angiogenesis. ${ }^{27}$

There are still not enough to uncover the black box of tumor metastasis. ${ }^{28-31}$ Most research on tumor metastasis has focused on the movement of tumor cells. ${ }^{32,33}$ It is generally regarded that chemokine signaling pathways promote chemotactic migration. Stuelten describes that when chemokines bind to their receptor, a series of downstream pathways will be activated. ${ }^{34}$ These include several classical pro-metastatic pathways, such as the PI3K and PTEN pathways. ${ }^{34}$ With that pathways being activated, cell redistributes the phosphatidylinositol-3,4,5-trisphosphate (PIP3) to the leading edge of cells and harbour 


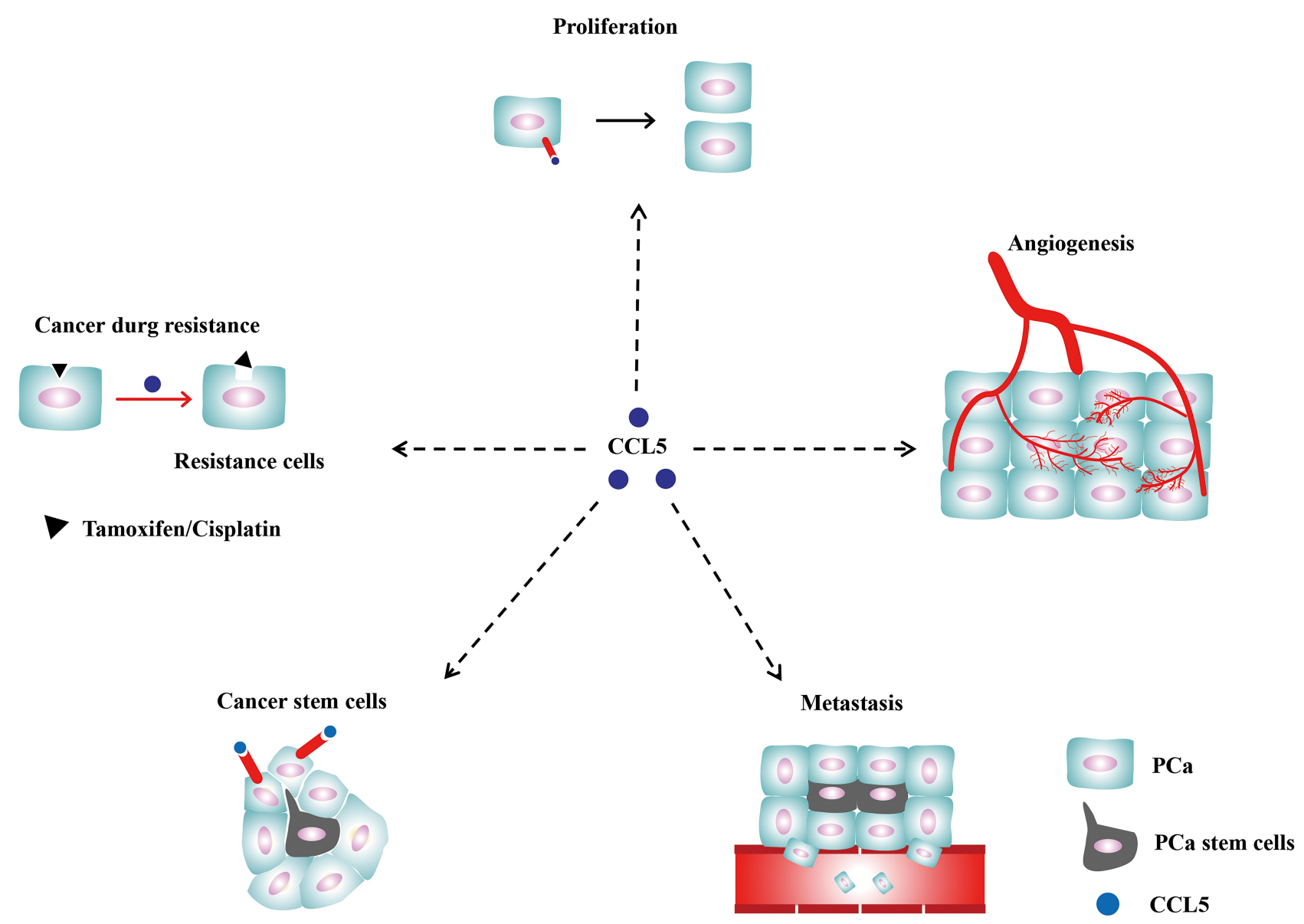

Figure I The role of CCL5 in PCa. CCL5 promotes PCa proliferation, angiogenesis, metastasis, the formation of stem cells and drug resistance via the crosslinking with CCR5 or CCRI.

pleckstrin homology $(\mathrm{PH})$ domain-containing proteins to the front of cells, and these changes cause actomyosin contraction and tail retraction and finally cause cell migration. ${ }^{23,34,35}$

CCL5, an important chemokine, promotes the migration and invasion of a variety of tumors, including PCa. ${ }^{36-41}$ As early as 2003, Vaday et al demonstrated that the invasive ability of PCa cells in the CCL5 experimental group was approximately twice as high as that in the control group, and the effect was weakened after administration of the CCL5 antagonist TAK-779. ${ }^{23}$ When CCL5 binds to CCR5, as described above, their interaction could also activate the PI3K/Akt pathway, which belongs to the star complex molecular pathway of PI3K-PTENmTOR, and the NF- $\mathrm{kB}$ pathway, which could promote the secretion of MMP-9 and MMP-2. ${ }^{34,42}$ In addition, the interaction between CCL5 and CCR5 could also promote metastasis by activating ERK and Rac signaling. ${ }^{34}$ Notably, Ras oncogenic signaling pathways could activate Jnk signaling pathways, which have been regarded as important signaling pathways in human tumor metastasis. ${ }^{34}$ And the activated Jnk signaling pathways not only leads to tumor cell proliferation but also promotes tumor metastasis by enhancing the expression of matrix metalloproteinases (MMPs) and reducing the expression of epithelial-mesenchymal transition (EMT) markers such as E-cadherin. ${ }^{43}$ Moreover, CCL5 could also upregulate MMP-9 by activating STAT3 signaling pathways. ${ }^{44}$ In other cases, the CCL5/CCR5 axis could promote tumor metastasis by activating MEK or ERK signaling pathways, leading to the activation of $\alpha v \beta 3$ integrin. ${ }^{45}$ Sottnik et al demonstrated that CCL5 derived from a mouse long bone microenvironment could promote metastasis of $\mathrm{PCa}$ cells and post-metastasis tumor growth. ${ }^{46}$ Similarly, Yeh found that cancer-associated fibroblasts (CAF) can secrete IL-6 and CCL5 to recruit macrophages, and then macrophages can promote $\mathrm{PCa}$ cells metastasis by secreting IL-6 in the tumor microenvironment. ${ }^{47} \mathrm{Zhao}^{48}$ illuminated that after co-culture of human umbilical vein endothelial cells (HUVECs) and the CRPC cell line C4-2, CCL5 was the 
most abundant chemokine in the culture supernatant, and it could enhance autophagy by inhibiting the AR pathway, ultimately reducing cell adhesion and promoting the metastasis of $\mathrm{PCa}^{48}$ Interestingly, another study found that CCL5 secreted by bone stromal cells could activate the AR signaling pathway which promoting metastasis of PCa cells. ${ }^{49}$ Karnoub found that CCL5 derived from mesenchymal stem cells could induce a metastatic phenotype and promote tumor metastasis by binding to its receptor CCR5 in primary tumor tissues. ${ }^{41}$ Furthermore, researchers have noted that distant tumor metastasis is closely related to the intravascular microenvironment, which is the result of the interaction between tumor cells, white blood cells, platelets, and vascular endothelial cells. $^{50}$ The study finally confirmed that tumor cells could interact with white blood cells and platelets, which could stimulate the microvascular endothelial cells to secrete CCL5, recruiting macrophages to promote tumor metastasis. ${ }^{50}$ In this study, CCL5 promoted tumor metastasis by changing the tumor metastasis microenvironment rather than directly affecting tumor cells. ${ }^{50}$ As mentioned earlier, CCL5 can bind to several different receptors and it plays a role in promoting tumor metastasis not only by binding to the major receptor CCR5 but also by binding to the receptor CCR1. ${ }^{18}$ Interaction between CCR1 and CCL5 could also promote the migration of taxaneresistant prostate cancer cells by activating Rac signaling pathway and by increasing the release of MMP-2 and MMP-9 via the activation of ERK signaling pathway, indicating that CCR1 could be a novel therapeutic target for taxane-resistant prostate cancer. ${ }^{15}$ Notably, prostate cancer stem cells (PCSCs) play a vital role in PCa metastasis, which is considered as a barrier for successful $\mathrm{PCa}$ treatment. For example, Huang demonstrated that CCL5 could promote $\mathrm{PCa}$ metastasis via activating $\beta$-catenin /STAT3 signaling which is a robustly relevant pathway of cancer stem cells. ${ }^{5}$

At present, the mainstream understanding is that CSCs is small fractions, approximately $0.1 \%$ to $1 \%$, of tumor cells in tumor tissues, with self-renewal and multi-directional differentiation capabilities. ${ }^{51}$ There is increasing evidence that chemokines could promote the self-renewal of CSCs. ${ }^{52-55}$ For example, CCL5 could increase the self-renewal of ovarian cancer stem-like cells. ${ }^{56}$ Studies have found that CCL5 secreted by PC3 and DU145 cells recruited bone marrow mesenchymal stem cells, constituting the tumor immune microenvironment of $\mathrm{PCa}$ which promoted the growth, metastasis, and drug resistance of PCa cells. ${ }^{57}$ Luo et al, via co-culture of bone marrow mesenchymal stem cells and PCa cells, confirmed a new pathway of action named the CCL5-AR-CXCR4/ZEB-1 axis. $^{58}$ The CCL5 mRNA expression and the secretion of CCL5 were upregulated, which could inhibit AR nuclear translocation and promote the self-renewal of PCSCs. ${ }^{58}$ Consequently, the activated axis led to the upregulation of ZEB-1, snail and MMP9 and finally promoted the metastasis of $\mathrm{PCa}^{58}$ Luo et al also showed that CCL5, released by bone marrow mesenchymal stem cells (BM-MSCs), enhanced hypoxia-inducible factor $2 \alpha$ (HIF2 $\alpha$ ) expression to inhibit AR and HSP90 binding, suppressing nuclear translocation of AR and silencing the androgen receptor (AR) signaling pathways that promote the self-renewal of PCSCs and PCa metastasis. ${ }^{59}$

In addition, it is necessary to determine the mechanism of tumor drug resistance, which can guide future clinical treatment strategies. When the TLR3 signaling pathway is triggered by DAMPs in HNSCC cells, downstream chemokines, such as CCL5, are activated resulting in resistance to cisplatin. ${ }^{60}$ In malignant ovarian cancer, cisplatin promoted the secretion of CCL5 by activating cancerassociated fibroblasts (CAFs), and then attenuated the cytotoxic effect of cisplatin on tumor cells. ${ }^{61}$ It was also found that CCL5 facilitated cisplatin resistance by promoting STAT3 phosphorylation and activating the STAT3 signaling pathway. ${ }^{61}$ CCL5 could also inhibit apoptosis signaling and promote tamoxifen resistance in breast cancer by promoting STAT3 phosphorylation. ${ }^{62}$ A study suggested that the treatment with docetaxel promotes the infiltration of $\mathrm{CD}^{+} \mathrm{T}$ cells in $\mathrm{PCa}$ tissue, and $\mathrm{CD}^{+}$ T cells secrete CCL5 to activate STAT3 signaling pathway which promotes PCa Docetaxel resistance. ${ }^{63}$ CCL5 has been shown to be associated with drug resistance in various tumors. Docetaxel resistance in advanced $\mathrm{PCa}$ is a significant factor in treatment failure. So, it is essential to confirm the key role of CCL5 in PCa drug resistance.

\section{The Therapeutic Effect of Drugs on the Action of CCL5/CCR5 Axis in Prostate Cancer}

Clinical evidence has indicated that the level of CCL5 in tissues or blood is associated with the poor prognosis of various tumors, including $\mathrm{PCa},{ }^{5}$ breast cancer, ${ }^{64}$ gastric cancer $^{65}$ and so on. By analysing 36 cases of $\mathrm{PCa}$ or benign prostatic hyperplasia, König et al identified that CCR5 is elevated in more than $89 \%$ of PCa tissues. ${ }^{17}$ Using GO term analysis, Sicoli et al found that the 
expression of CCR5 in mouse PCa tissue was 11.3 times higher than that in mouse normal prostate tissue. ${ }^{66}$ In addition, by consulting the Oncomine database, they found that the expression of CCR5 in patients with $\mathrm{PCa}$ was 4 to 5 times higher than that in normal controls, which can also be confirmed by other public databases. ${ }^{66}$ Through the analysis of clinical specimens of patients, a study found that in the androgen-independent prostate cancer (AIPC) bone metastasis site, the expression of CCL5 was approximately 24 times higher than that of the primary site of $\mathrm{PCa}{ }^{13}$ Likewise, through analysis of the difference between tumor and non-neoplastic tissues in patients with $\mathrm{PCa}$, several studies demonstrated that the expression of CCL5 significantly increased in stromal cells $^{67}$ and was approximately 2.31 times than that of normal adjacent tissues. ${ }^{68}$ According to the analysis of 272 clinical serum specimens, the level of CCL5 in patients with cancerous prostates was approximately $25,323.63 \pm 1210.77 \mathrm{pg} / \mathrm{mL}$, which was different from that of patients with non-cancerous prostates as $22,339.28 \pm 1185.27 \mathrm{pg} / \mathrm{mL}(P=0.080) .{ }^{69}$ These findings suggest that the CCL5/CCR5 axis has good clinical application prospects as a new therapeutic target and for monitoring or predicting disease development in liquid biopsy. Evaluated in germ-line DNA samples from 814 AfricanAmerican and Jamaican men by genetic analysis of variant chemokine-associated SNPs, for example, CCR5 gene was linked with a $1.52-1.73$ fold increase in PCa risk. ${ }^{70}$ CCL5/ CCR5 inhibitors could exert their antitumor effects. ${ }^{21,66}$

The current drug development focused on the CCL5/ CCR5 axis is primarily directed at CCR5. And the importance of CCR5 in HIV infection has led to the development of compounds that target CCR5. ${ }^{71}$ Currently, Maraviroc, a marketed drug for CCR5, is primarily used to treat HIV because of its good tolerance, and it is also beneficial in advanced metastatic colorectal cancer. $^{72}$ As seen from various studies and reviews, antagonists of CCR5 in previous clinical applications are vicriviroc, ${ }^{73}$ aplaviroc, ${ }^{74}$ INCB009471, ${ }^{75}$ cenicriviroc (also known as tako-799), ${ }^{76}$ PRO140 (Leronlimab), ${ }^{77}$ anibamine, ${ }^{78}$ Met-CCL5, ${ }^{79}$ OTR $4120^{80}$ and OTR4131, ${ }^{80}$ DT- $13,{ }^{81}$ and Cenicriviroc TBR- $652 .{ }^{82}$ Herein, the following will focus on CCR5 inhibitors associated with PCa, including cenicriviroc, maraviroc, anibamine and DT-13 which are shown in Table 1.

\section{Maraviroc}

Maraviroc, the first-in-class CCR5 inhibitor, was approved by the FDA in 2007 and is used primarily for the clinical treatment of $\mathrm{HIV}{ }^{83}$ with a $50 \%$ inhibitory concentration (IC50) of CCR5 of $5.2 \mathrm{nM}^{84}$ Increased expression of CCL5 and CCR5 is associated with metastasis and poor prognosis in $\mathrm{PCa} .{ }^{5}$ Building a stable animal model of bone metastasis contributes to the research of PCa. Sicoli have innovatively established a new stable cell line for the study of PCa bone metastases. ${ }^{66}$ They utilized v-Src oncogenetransformed prostate epithelial cells and then used these cells to construct a mouse metastasis model, finding that metastasis occurred in all mice. ${ }^{66}$ They demonstrated that there was a significant difference in the expression of CCR5 between normal mouse prostate tissue and v-SrcPEC subcutaneously implanted tumor tissue. ${ }^{66}$ At the same time, Src is activated in AKT survival pathway, and is required for CXCLR activation in metastatic cancer,

Table I The Therapeutic Effect of Drugs on the Action of CCL5/CCR5 Axis in Prostate Cancer

\begin{tabular}{|c|c|c|c|}
\hline Name & Characteristics & Effects of Anti-Prostate Cancer & References \\
\hline Maraviroc & $\begin{array}{l}\text { 1. CCR5 Antagonist. } \\
\text { 2. IC50 of CCR5 is } 5.2 \mathrm{nM} \text {. }\end{array}$ & $\begin{array}{l}\text { I. Reduced the rate of brain metastasis and bone metastasis } \\
\text { in vivo. }\end{array}$ & {$[66,83,84]$} \\
\hline $\begin{array}{l}\text { Cenicriviroc (also known as } \\
\text { TAK-799) }\end{array}$ & $\begin{array}{l}\text { I.CCR5 and CCR2 } \\
\text { Antagonist. } \\
2 . I C 50 \text { of CCR5 is } 1.4 \mathrm{nM} \text {. }\end{array}$ & I.Inhibition of tumor proliferation and migration in vitro. & {$[21,76]$} \\
\hline Anibamine & $\begin{array}{l}\text { I.CCR5 Antagonist. } \\
2.1 \text {. } 50 \text { of CCR5 is } 1.0 \mu \mathrm{M} \text {. }\end{array}$ & $\begin{array}{l}\text { I.Inhibition of tumor proliferation and migration in vitro. } \\
\text { 2.provides a structural skeleton of chemical modification of } \\
\text { CCR5 antagonist in vitro. }\end{array}$ & [85-88] \\
\hline DT-I3 & $\begin{array}{l}\text { I.CCR5 Antagonist. } \\
2.1 \text {. } 50 \text { of CCR5 is } 1.0 \mu \mathrm{M} \text {. }\end{array}$ & $\begin{array}{l}\text { I.Inhibition of tumor proliferation, angiogenesis and migration } \\
\text { in vitro. } \\
\text { 2.Synergistically enhance the effect of chemotherapeutics in vitro. }\end{array}$ & {$[81,90-93]$} \\
\hline
\end{tabular}


maraviroc prostate cancer metastasis to bone by block CCR5 signaling. ${ }^{66}$

\section{Cenicriviroc}

Cenicriviroc, also called TAK-799, is a CCR5 non-peptide small molecule inhibitor that was first reported in a study on HIV in 1999. With good oral bioavailability and long half-life, its 50\% inhibitory concentration (IC50) of CCR5 is $1.4 \mathrm{nM}^{76}$ As early as $2006^{21}$, Dr. Vaday found that TAK-799 attenuated the effect of CCL5 in promoting migration and proliferation of PCa cell lines DU145 and PC3, but the use of TAK-799 alone had no inhibitory effect, which means that other experiments, such as experiments in vivo, are needed to confirm whether it has an effect on $\mathrm{PCa}{ }^{21}$

\section{Anibamine}

Anibamine, the natural product CCR5 antagonist with an IC50 at $1 \mu \mathrm{M}$ in competition with ${ }^{125} \mathrm{I}$-gp120, plays a decent role against $\mathrm{PCa}$ and provides a structural skeleton for chemical modification of the CCR5 antagonist. ${ }^{85}$ Studies have found that anibamine could significantly inhibit the proliferation and metastasis of PCa cells by inhibiting CCR5. ${ }^{86,87}$ Under the full understanding of the structural skeleton of anibamine, Zhang et al constructed 17 anibamine analogues and tested their activity, enhancing the anti-tumor activity of anibamine through structural modification and showing potential for good clinical application of an anibamine antagonist in $\mathrm{PCa}{ }^{88}$

\section{DT-I 3}

DT-13, as a saponin monomer of dwarf lilyturf tuber, ${ }^{89}$ has chemical properties and good oral bioavailability compared with similar drugs and inhibits the expression of CCR5 as well as the secretion of CCL5. ${ }^{90,91}$ A study found that DT-13 has a very comprehensive anti-tumor activity, inhibiting the proliferation, metastasis, and angiogenesis of tumor cells. ${ }^{92}$ In addition, DT-13 could also augment the role of other antitumor drugs and synergistically enhance the effect of vinorelbine in non-small cell lung cancer ${ }^{92}$ and the effect of topotecan in gastric cancer, ${ }^{93}$ for example. The IC50 of PCa cell proliferation inhibition by DT-13 is approximately $5 \mu \mathrm{M} .{ }^{81}$ Other studies have shown that DT-13 can inhibit the expression of Integrin $\beta 1$ and MMP2/9 and induce apoptosis by blocking the PI3K/Akt pathway, thereby inhibiting the proliferation and migration of PCa cells. ${ }^{81}$

\section{Regulation of CCL5 Expression and Its Downstream Signaling Pathways The Transcription Factor Related to CCL5 Expression}

The full length of CCL5 gene is $9303 \mathrm{bp}$, and it is located on chromosome $17 \mathrm{q} 12$ by searching in NCBI database. ${ }^{18}$ It has three transcripts: the 1365 bp CCL5201, the 1244 bp CCL5-202 and the 719 bp CCL5-203. The transcription factors regulating human CCL5 expression are complex and diverse. Current findings indicated that the CCL5 promoter submodels can be divided into six regions. ${ }^{94,95}$ Based on previous studies, CCL5 promoter base sequence and binding transcription factor were summarized ${ }^{94,95}$ and shown in Figure 2. Moreover, there are some bioinformatic strategies to predict the transcription factor of CCL5. Firstly, the base sequence $-2000 \mathrm{bp}$ to $100 \mathrm{bp}$ from the CCL5 transcriptional start point was found by analyzing the

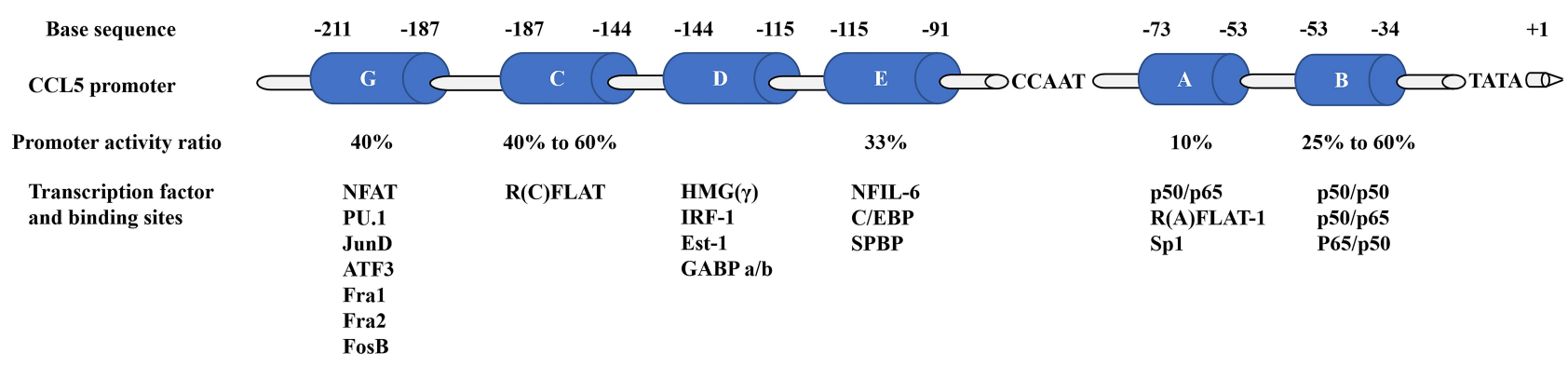

Figure 2 Model of the regions of the human CCL5 promoter. The regions A to E and G named in order of discovery and their base sequence and binding transcription factor were summarized. The percentages below each region demonstrate the percentage of CCL5 promoter activity remaining after deleting that region.

Abbreviations: NFAT, nuclear factor of activated T cells; PU.I/Ets-I, Ets family members; ATF3, activating transcription factor 3; JunD/FosB/Fra I/Fra2, API family members; $\mathrm{R}(\mathrm{C}) \mathrm{FLAT}, \mathrm{R}(\mathrm{C})$ factor of late activated T cells; HMG $(\gamma)$, high-mobility group protein ( $\gamma$ ); IRF-I, interferon regulatory factor-I; GABP, GA binding protein; NF-IL6, nuclear factor of interleukin 6; C/EBP, CAAT/enhancer binding protein; SPBP, stromelysin-I PDGF responsive element binding protein; NF-kB, nuclear factor kappa B; p50/p50, NFKB p50 subunit homodimer; p50/p65, NF-KB Rel family members; R(A)FLAT-I, R(A) factor of late activated T cells-I; SpI, stimulating protein I. 


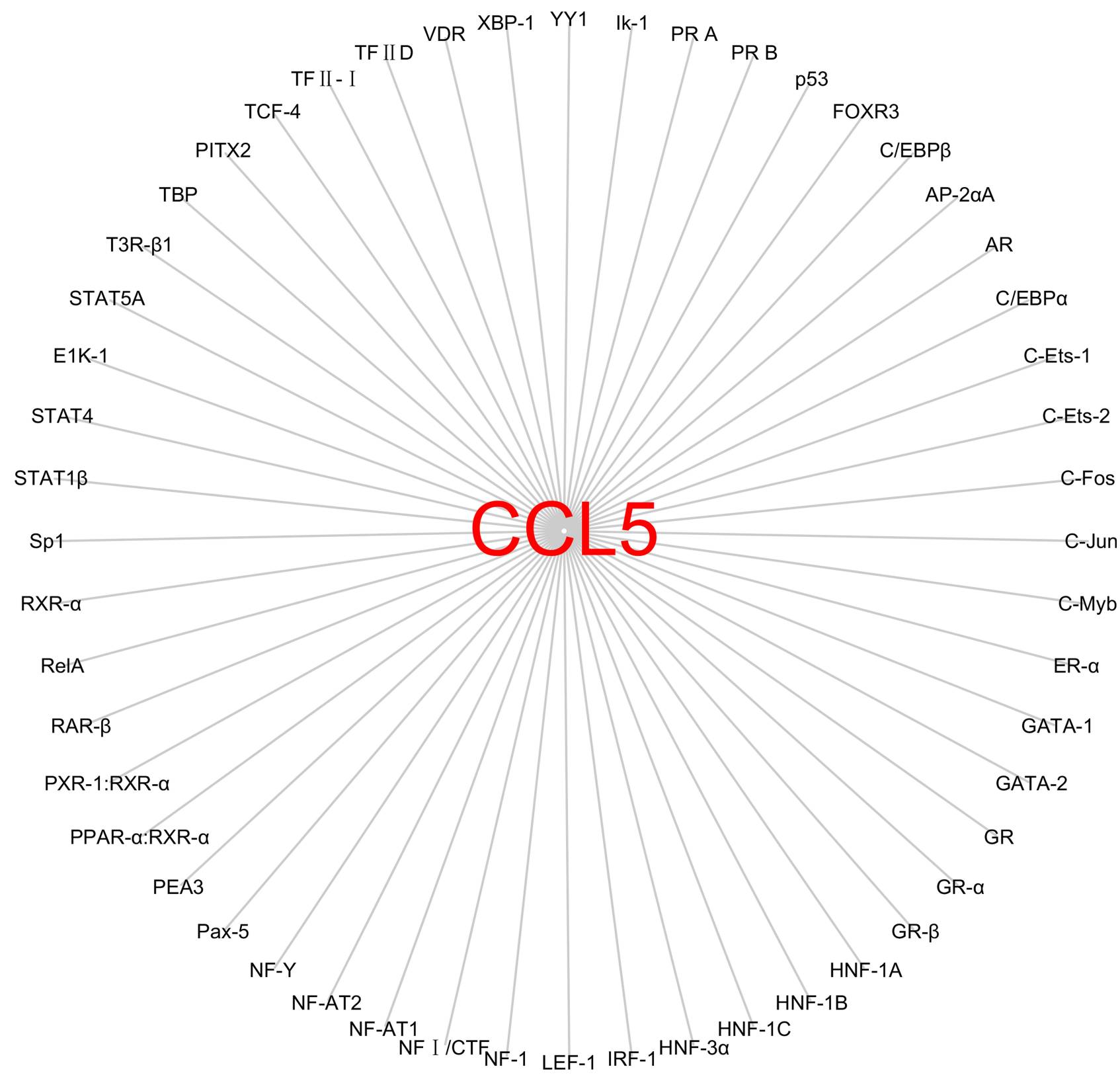

Figure 3 The transcription factor regulating CCL5 predicted by analysing the UCSC database. We firstly found the base sequence -2000 bp to 100 bp from the CCL5 transcriptional start point and then predicted the transcription factor with the base sequence through using the PROMO database with $5 \%$ fault tolerance.

Abbreviations: TBP, TATA-binding protein; TFII D, general transcription factor; PITX2, paired-liked homeodo-main transcription factor 2; TCF-4, T cell factor 4; VDR, vitamin D receptor; XBP-I, X-box binding protein-I; YYI, YIN-YANG I; IK-I, IKaros-I; PR, progesterone receptor; P53, tumor suppressor gene; FOX, Forkhead box; C/ EBP, CAAT/enhancer binding protein; AP-2, integrase-type DNA-binding superfamily protein; AR, androgen receptor; Jun/Fos, API family members; Ets-1/Ets-2, Ets family members; C-Myb, MYB proto-oncogene; ER, Leucine-rich receptor-like protein kinase family protein; GATA, Glutamyl-tRNA amidotransferase, subunit A; GR, glutathione reductase; HNF-IA/HNF-IB/HNF-IC, HNFI homeobox family members; IRF-I, interferon regulatory factor-I; LEF-I, lymphoid enhancer binding factor I; NF-I, nuclear factor-I; NF-AT, nuclear factor of activated T cells; NFI/CTF, nuclear factor I family of site-specific DNA-binding proteins; NF-Y, nuclear factor-Y; Pax-5, paired box 5; PEA3, polymoma virus enhancer 3; PPAR, peroxisome proliferator-activated receptor; PXR/RAR/RXR, nuclear receptor; RelA, RELA proto-oncogene, NF-kB subunit; SpI, stimulating protein I; STAT, signal transducer and activator of transcription; T3R- $\beta$ I, thyroid hormone receptor-beta I.

UCSC database. Secondly, based on the sequence of CCL5 transcriptional start point, the transcription factor regulating CCL5 was predicted through using the PROMO database with $5 \%$ fault tolerance, which are shown in Figure 3 plotted via Cytoscape software. ${ }^{96}$

\section{The Downstream Pathways of Chemokine CCL5}

First, CCL 5 can upregulate cyclin D1 expression by promoting phosphorylation of STAT5, which leads to the proliferation of PCa cells. ${ }^{22}$ Second, CCL5 can promote ovarian cancer 


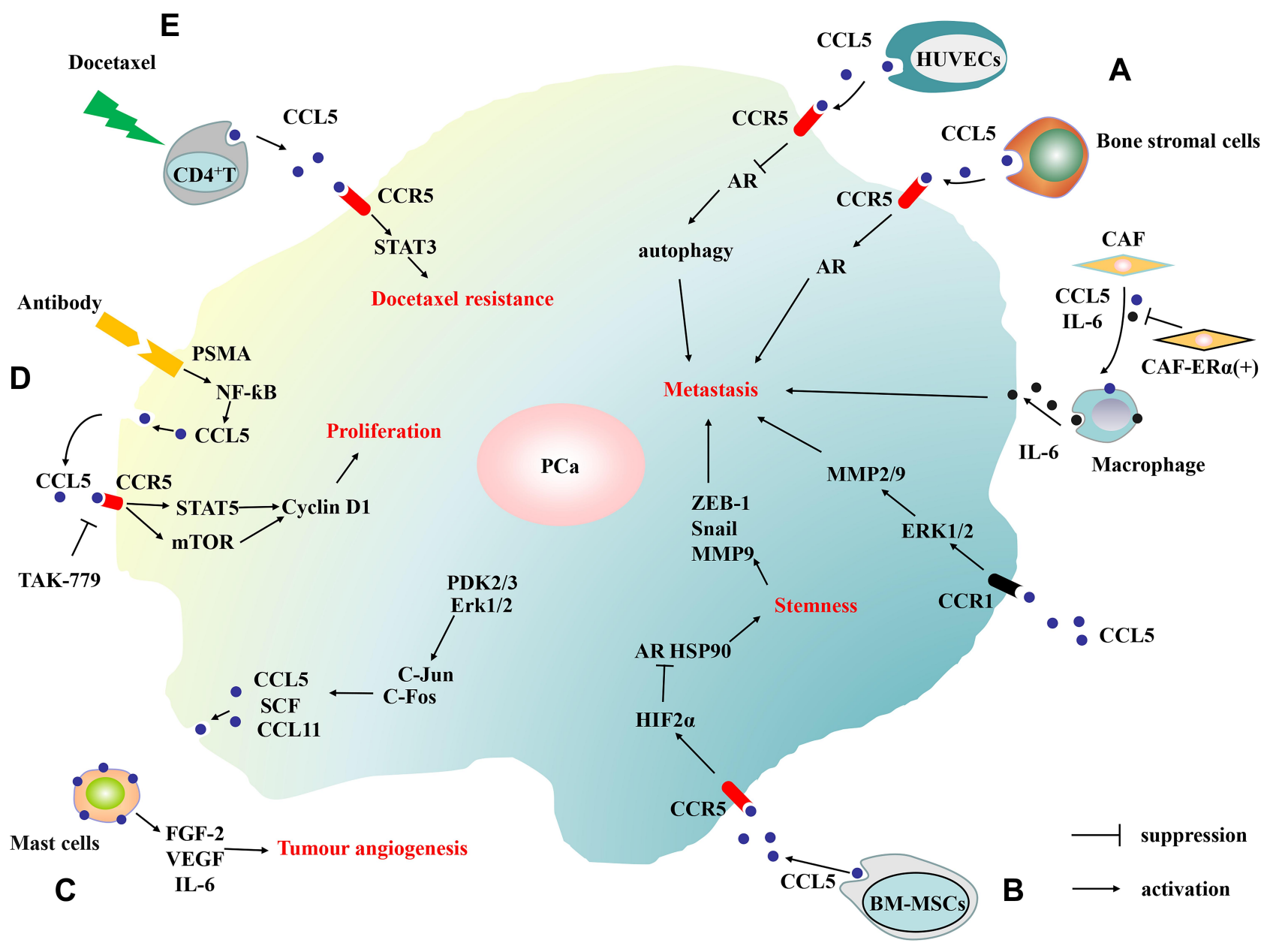

Figure 4 The schematic diagram of the downstream pathways of CCL5 in PCa. (A) The human umbilical vein endothelial cells (HUVECs), bone stromal cells, macrophages and cancer-associated fibroblasts (CAF) can promote PCa cell metastasis by secreting CCL5 in tumor microenvironment. (B) By secreting CCL5, bone marrow mesenchymal stem cells (BM-MSCs) promote PCa metastasis and stemness. (C) By secreting CCL5, PCa recruit mast cells which can release FGF-2, VEGF and IL-6 to promote angiogenesis. (D) CCL5 promotes the proliferation of prostate cancer. (E) CCL5 promotes the drug resistance of prostate cancer.

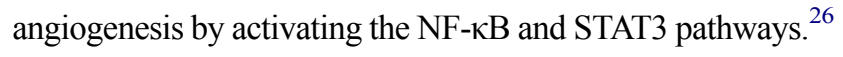
Luo et al demonstrated that the CCL5/HIF2 $\alpha / \mathrm{AR} / \mathrm{ZEB}-1$ axis promotes the self-renewal of PCSCs and PCa metastasis. ${ }^{58,59}$ CCL5 enhanced hypoxia-inducible factor $2 \alpha$ (HIF2 $\alpha$ ) expression to inhibit AR and HSP90 binding, thereby decreasing the nuclear translocation of AR, which ultimately leads to the upregulation of ZEB-1. ${ }^{59}$ Third, Zhao et al illuminated that CCL5 can increase autophagy, which can weaken cell adhesion and further the metastasis of $\mathrm{PCa} .{ }^{48}$ Kato found that the crosslinking of CCL5 and CCR1 activates the RK signaling pathway to promote the release of MMP2/9 and activate the Rac signaling pathway, ultimately facilitate PCa metastasis. ${ }^{15}$ Moreover, Xiang found that CCL5 can activate STAT3 signaling pathway and that contributes to $\mathrm{PCa}$ docetaxel resistance. ${ }^{63}$ These are summarized and shown in Figure 4.

\section{Conclusion}

This review clarifies the role of CCL5 in PCa and the development made in CCL5 research. The chemokine CCL5 activates a series of downstream pathways through specific binding to the CCR5 receptor. CCL5 plays an important role in tumor growth, metastasis, angiogenesis, tumor resistance, and self-renewal of tumor stem cells. As a key factor that promotes tumorigenesis and tumor development, CCL5 is regulated by multiple elements. Some drugs can reduce the expression of CCR5 and the secretion of CCL5, thus exerting effective antitumor activity. The CCL5/CCR5 axis has a quite broad tumor-promoting effect. And its antagonists are prospective for clinical application which is worthy of further study. 


\section{Funding}

This work is supported by the National Natural Science Foundation of China (No. 81774067) and Specific Research Fund for TCM Science and Technology of Guangdong Provincial Hospital of Chinese Medicine (YN2016MJ03).

\section{Disclosure}

The authors report no conflicts of interest in this work.

\section{References}

1. Siegel RL, Miller KD, Jemal A. Cancer statistics, 2019. CA Cancer J Clin. 2019;69(1):7-34.

2. Nagarsheth N, Wicha MS, Zou W. Chemokines in the cancer microenvironment and their relevance in cancer immunotherapy. Nat Rev Immunol. 2017;17(9):559-572.

3. Ben-Baruch A. The multifaceted roles of chemokines in malignancy. Cancer Metastasis Rev. 2006;25(3):357-371.

4. Salazar N, Castellan M, Shirodkar SS, Lokeshwar BL. Chemokines and chemokine receptors as promoters of prostate cancer growth and progression. Crit Rev Eukaryot Gene Expr. 2013;23(1):77-91.

5. Huang R, Wang S, Wang $\mathrm{N}$, et al. CCL5 derived from tumor-associated macrophages promotes prostate cancer stem cells and metastasis via activating beta-catenin/STAT3 signaling. Cell Death Dis. 2020;11(4):234.

6. Ali S, Lazennec G. Chemokines: novel targets for breast cancer metastasis. Cancer Metastasis Rev. 2007;26(3-4):401-420.

7. Griffith JW, Sokol CL, Luster AD. Chemokines and chemokine receptors: positioning cells for host defense and immunity. Annu Rev Immunol. 2014;32:659-702.

8. Marra F, Tacke F. Roles for chemokines in liver disease. Gastroenterology. 2014;147(3):577-594.e571

9. Walz DA, Wu VY, de Lamo R, Dene H, McCoy LE. Primary structure of human platelet factor 4. Thromb Res. 1977;11 (6):893-898.

10. Gueron G, De Siervi A, Vazquez E. Advanced prostate cancer: reinforcing the strings between inflammation and the metastatic behavior. Prostate Cancer Prostatic Dis. 2012;15(3):213-221.

11. Chow MT, Luster AD. Chemokines in cancer. Cancer Immunology Res. 2014;2(12):1125-1131.

12. Christopherson K, Hromas R. Chemokine regulation of normal and pathologic immune responses. Stem Cells. 2001;19(5):388-396.

13. Maolake A, Izumi K, Shigehara K, et al. Tumor-associated macrophages promote prostate cancer migration through activation of the CCL22-CCR4 axis. Oncotarget. 2017;8(6):9739-9751.

14. Sung SY, Hsieh CL, Law A, et al. Coevolution of prostate cancer and bone stroma in three-dimensional coculture: implications for cancer growth and metastasis. Cancer Res. 2008;68(23):9996-10003.

15. Kato T, Fujita Y, Nakane K, et al. CCR1/CCL5 interaction promotes invasion of taxane-resistant $\mathrm{PC} 3$ prostate cancer cells by increasing secretion of MMPs 2/9 and by activating ERK and Rac signaling. Cytokine. 2013;64(1):251-257.

16. Schall TJ, Jongstra J, Dyer BJ, et al. A human T cell-specific molecule is a member of a new gene family. J Immunol. 1988;141 (3):1018-1025.

17. König JE, Senge T, Allhoff EP, König W. Analysis of the inflammatory network in benign prostate hyperplasia and prostate cancer Prostate. 2004;58(2):121-129.

18. Soria G, Ben-Baruch A. The inflammatory chemokines CCL2 and CCL5 in breast cancer. Cancer Lett. 2008;267(2):271-285.
19. Velasco-Velazquez M, Xolalpa W, Pestell RG. The potential to target CCL5/CCR5 in breast cancer. Expert Opin Ther Targets. 2014;18 (11):1265-1275.

20. Marques RE, Guabiraba R, Russo RC, Teixeira MM. Targeting CCL5 in inflammation. Expert Opin Ther Targets. 2013;17(12):1439-1460.

21. Vaday GG, Peehl DM, Kadam PA, Lawrence DM. Expression of CCL5 (RANTES) and CCR5 in prostate cancer. The Prostate. 2006;66(2):124-134.

22. Colombatti M, Grasso S, Porzia A, et al. The prostate specific membrane antigen regulates the expression of IL-6 and CCL5 in prostate tumour cells by activating the MAPK pathways. PLoS One. 2009;4(2):e4608.

23. Aldinucci D, Colombatti A. The inflammatory chemokine CCL5 and cancer progression. Mediators Inflamm. 2014;2014:292376.

24. Barcelos LS, Coelho AM, Russo RC, et al. Role of the chemokines CCL3/MIP-1 alpha and CCL5/RANTES in sponge-induced inflammatory angiogenesis in mice. Microvasc Res. 2009;78(2):148-154.

25. Ambati BK, Anand A, Joussen AM, Kuziel WA, Adamis AP, Ambati J. Sustained inhibition of corneal neovascularization by genetic ablation of CCR5. Invest Ophthalmol Vis Sci. 2003;44 (2):590-593.

26. Tang S, Xiang T, Huang S, et al. Ovarian cancer stem-like cells differentiate into endothelial cells and participate in tumor angiogenesis through autocrine CCL5 signaling. Cancer Lett. 2016;376 (1):137-147.

27. Katona TM, Neubauer BL, Iversen PW, Zhang S, Baldridge LA, Cheng L. Elevated expression of angiogenin in prostate cancer and its precursors. Clin Cancer Res. 2005;11(23):8358-8363.

28. Bernards R, Weinberg RA. A progression puzzle. Nature. 2002;418 (6900):823.

29. Meehan WJ, Samant RS, Hopper JE, et al. Breast cancer metastasis suppressor 1 (BRMS1) forms complexes with retinoblastoma-binding protein 1 (RBP1) and the mSin3 histone deacetylase complex and represses transcription. $J$ Biol Chem. 2004;279(2):1562-1569.

30. Horak CE, Mendoza A, Vega-Valle E, et al. Nm23-H1 suppresses metastasis by inhibiting expression of the lysophosphatidic acid receptor EDG2. Cancer Res. 2007;67(24):11751-11759.

31. Sahni S, Bae DH, Lane DJ, et al. The metastasis suppressor, N-myc downstream-regulated gene 1 (NDRG1), inhibits stress-induced autophagy in cancer cells. $J$ Biol Chem. 2014;289(14):9692-9709.

32. Kalluri R, Weinberg RA. The basics of epithelial-mesenchymal transition. J Clin Invest. 2009;119(6):1420-1428.

33. Tsuji T, Ibaragi S, Hu GF. Epithelial-mesenchymal transition and cell cooperativity in metastasis. Cancer Res. 2009;69(18):7135-7139.

34. Stuelten CH, Parent CA, Montell DJ. Cell motility in cancer invasion and metastasis: insights from simple model organisms. Nat Rev Cancer. 2018;18(5):296-312.

35. Ridley AJ, Schwartz MA, Burridge K, et al. Cell migration: integrating signals from front to back. Science. 2003;302(5651):1704-1709.

36. Azenshtein E, Luboshits G, Shina S, et al. The CC chemokine RANTES in breast carcinoma progression: regulation of expression and potential mechanisms of promalignant activity. Cancer Res. 2002;62(4):1093-1102.

37. Robinson SC, Scott KA, Balkwill FR. Chemokine stimulation of monocyte matrix metalloproteinase-9 requires endogenous TNF-alpha. Eur J Immunol. 2002;32(2):404-412.

38. Long H, Xiang T, Qi W, et al. CD133+ ovarian cancer stem-like cells promote non-stem cancer cell metastasis via CCL5 induced epithelial-mesenchymal transition. Oncotarget. 2015;6(8):5846-5859.

39. Huang CY, Fong YC, Lee CY, et al. CCL5 increases lung cancer migration via PI3K, Akt and NF-kappaB pathways. Biochem Pharmacol. 2009;77(5):794-803.

40. Long H, Xie R, Xiang T, et al. Autocrine CCL5 signaling promotes invasion and migration of CD133+ ovarian cancer stem-like cells via NF-kappaB-mediated MMP-9 upregulation. Stem Cells. 2012;30 (10):2309-2319. 
41. Karnoub AE, Dash AB, Vo AP, et al. Mesenchymal stem cells within tumour stroma promote breast cancer metastasis. Nature. 2007;449 (7162):557-563.

42. Hawkins PT, Stephens LR. PI3K signalling in inflammation. Biochim Biophys Acta. 2015;1851(6):882-897.

43. Chen F. JNK-induced apoptosis, compensatory growth, and cancer stem cells. Cancer Res. 2012;72(2):379-386.

44. Kim JE, Kim HS, Shin YJ, et al. LYR71, a derivative of trimeric resveratrol, inhibits tumorigenesis by blocking STAT3-mediated matrix metalloproteinase 9 expression. Exp Mol Med. 2008;40(5):514-522.

45. Wang SW, Wu HH, Liu SC, et al. CCL5 and CCR5 interaction promotes cell motility in human osteosarcoma. PLoS One. 2012;7(4):e35101.

46. Sottnik JL, Dai J, Zhang H, Campbell B, Keller ET. Tumor-induced pressure in the bone microenvironment causes osteocytes to promote the growth of prostate cancer bone metastases. Cancer Res. 2015;75 (11):2151-2158

47. Yeh CR, Slavin S, Da J, et al. Estrogen receptor alpha in cancer associated fibroblasts suppresses prostate cancer invasion via reducing CCL5, IL6 and macrophage infiltration in the tumor microenvironment. Mol Cancer. 2016;15:7.

48. Zhao R, Bei X, Yang B, et al. Endothelial cells promote metastasis of prostate cancer by enhancing autophagy. J Exp Clin Cancer Res. 2018;37(1):221.

49. Urata S, Izumi K, Hiratsuka K, et al. C-C motif ligand 5 promotes migration of prostate cancer cells in the prostate cancer bone metastasis microenvironment. Cancer Sci. 2018;109(3):724-731.

50. Laubli H, Spanaus KS, Borsig L. Selectin-mediated activation of endothelial cells induces expression of CCL5 and promotes metastasis through recruitment of monocytes. Blood. 2009;114(20):4583-4591.

51. Batlle E, Clevers H. Cancer stem cells revisited. Nat Med. 2017;23 (10):1124-1134.

52. Ginestier C, Liu S, Diebel ME, et al. CXCR1 blockade selectively targets human breast cancer stem cells in vitro and in xenografts. $J$ Clin Invest. 2010;120(2):485-497.

53. Hermann PC, Huber SL, Herrler T, et al. Distinct populations of cancer stem cells determine tumor growth and metastatic activity in human pancreatic cancer. Cell Stem Cell. 2007;1(3):313-323.

54. Dewan MZ, Ahmed S, Iwasaki Y, Ohba K, Toi M, Yamamoto N. Stromal cell-derived factor-1 and CXCR4 receptor interaction in tumor growth and metastasis of breast cancer. Biomed Pharmacother. 2006;60(6):273-276.

55. Tsuyada A, Chow A, Wu J, et al. CCL2 mediates cross-talk between cancer cells and stromal fibroblasts that regulates breast cancer stem cells. Cancer Res. 2012;72(11):2768-2779.

56. Zou W, Wicha MS. Chemokines and cellular plasticity of ovarian cancer stem cells. Oncoscience. 2015;2(7):615-616.

57. Borghese C, Cattaruzza L, Pivetta E, et al. Gefitinib inhibits the cross-talk between mesenchymal stem cells and prostate cancer cells leading to tumor cell proliferation and inhibition of docetaxel activity. J Cell Biochem. 2013;114(5):1135-1144.

58. Luo J, Ok Lee S, Liang L, et al. Infiltrating bone marrow mesenchymal stem cells increase prostate cancer stem cell population and metastatic ability via secreting cytokines to suppress androgen receptor signaling. Oncogene. 2014;33(21):2768-2778.

59. Luo J, Lee SO, Cui Y, Yang R, Li L, Chang C. Infiltrating bone marrow mesenchymal stem cells (BM-MSCs) increase prostate cancer cell invasion via altering the CCL5/HIF2alpha/androgen receptor signals. Oncotarget. 2015;6(29):27555-27565.

60. Chuang HC, Chou MH, Chien CY, Chuang JH, Liu YL. Triggering TLR3 pathway promotes tumor growth and cisplatin resistance in head and neck cancer cells. Oral Oncol. 2018;86:141-149.

61. Zhou B, Sun C, Li N, et al. Cisplatin-induced CCL5 secretion from CAFs promotes cisplatin-resistance in ovarian cancer via regulation of the STAT3 and PI3K/Akt signaling pathways. Int J Oncol. 2016;48 (5):2087-2097.
62. Yi EH, Lee CS, Lee JK, et al. STAT3-RANTES autocrine signaling is essential for tamoxifen resistance in human breast cancer cells. Mol Cancer Res. 2013;11(1):31-42.

63. Xiang $\mathrm{P}$, Jin $\mathrm{S}$, Yang $\mathrm{Y}$, et al. Infiltrating CD4+ $\mathrm{T}$ cells attenuate chemotherapy sensitivity in prostate cancer via CCL5 signaling. The Prostate. 2019;79(9):1018-1031.

64. Gonzalez RM, Daly DS, Tan R, Marks JR, Zangar RC. Plasma biomarker profiles differ depending on breast cancer subtype but RANTES is consistently increased. Cancer Epidemiol Biomarkers Prevent. 2011;20(7):1543-1551.

65. Sugasawa H, Ichikura T, Tsujimoto H, et al. Prognostic significance of expression of CCL5/RANTES receptors in patients with gastric cancer. J Surg Oncol. 2008;97(5):445-450.

66. Sicoli D, Jiao X, Ju X, et al. CCR5 receptor antagonists block metastasis to bone of v-Src oncogene-transformed metastatic prostate cancer cell lines. Cancer Res. 2014;74(23):7103-7114.

67. Gregg JL, Brown KE, Mintz EM, Piontkivska H, Fraizer GC. Analysis of gene expression in prostate cancer epithelial and interstitial stromal cells using laser capture microdissection. BMC Cancer. 2010;10:165.

68. Tsaur I, Noack A, Makarevic J, et al. CCL2 Chemokine as a Potential Biomarker for Prostate Cancer: a Pilot Study. Cancer Res Treatment. 2015;47(2):306-312.

69. Agarwal M, He C, Siddiqui J, Wei JT, Macoska JA. CCL11 (eotaxin-1): a new diagnostic serum marker for prostate cancer. The Prostate. 2013;73(6):573-581.

70. Kidd LR, Jones DZ, Rogers EN, et al. Chemokine Ligand 5 (CCL5) and chemokine receptor (CCR5) genetic variants and prostate cancer risk among men of African Descent: a case-control study. Hered Cancer Clin Pract. 2012;10(1):16.

71. Zheng Y, Han GW, Abagyan R, et al. Structure of CC Chemokine Receptor 5 with a Potent Chemokine Antagonist Reveals Mechanisms of Chemokine Recognition and Molecular Mimicry by HIV. Immunity. 2017;46(6):1005-1017.e1005.

72. Halama N, Zoernig I, Berthel A, et al. Tumoral Immune Cell Exploitation in Colorectal Cancer Metastases Can Be Targeted Effectively by Anti-CCR5 Therapy in Cancer Patients. Cancer Cell. 2016;29(4):587-601.

73. Strizki JM, Tremblay C, Xu S, et al. Discovery and characterization of vicriviroc (SCH 417690), a CCR5 antagonist with potent activity against human immunodeficiency virus type 1. Antimicrob Agents Chemother. 2005;49(12):4911-4919.

74. Nichols WG, Steel HM, Bonny T, et al. Hepatotoxicity observed in clinical trials of aplaviroc (GW873140). Antimicrob Agents Chemother. 2008;52(3):858-865.

75. Stellbrink HJ. Novel compounds for the treatment of HIV type-1 infection. Antivir Chem Chemother. 2009;19(5):189-200.

76. Baba M, Nishimura O, Kanzaki N, et al. A small-molecule, nonpeptide CCR5 antagonist with highly potent and selective anti-HIV-1 activity. Proc Natl Acad Sci U S A. 1999;96(10):5698-5703.

77. Maeda K, Das D, Nakata H, Mitsuya H. CCR5 inhibitors: emergence, success, and challenges. Expert Opin Emerg Drugs. 2012;17 (2):135-145.

78. Haney KM, Zhang F, Arnatt CK, et al. The natural product CCR5 antagonist anibamine and its analogs as anti-prostate cancer agents. Bioorg Med Chem Lett. 2011;21(18):5159-5163.

79. Mack M, Cihak J, Simonis C, et al. Expression and characterization of the chemokine receptors CCR2 and CCR5 in mice. J Immunol. 2001;166(7):4697-4704.

80. Sutton A, Friand V, Papy-Garcia D, et al. Glycosaminoglycans and their synthetic mimetics inhibit RANTES-induced migration and invasion of human hepatoma cells. Mol Cancer Ther. 2007;6 (11):2948-2958.

81. Wang Z, Wang Y, Zhu S, et al. DT-13 Inhibits Proliferation and Metastasis of Human Prostate Cancer Cells Through Blocking PI3K/Akt Pathway. Front Pharmacol. 2018;9:1450. 
82. Aldinucci D, Casagrande N. Inhibition of the CCL5/CCR5 Axis against the Progression of Gastric Cancer. Int J Mol Sci. 2018;19:5.

83. Dorr P, Westby M, Dobbs S, et al. Maraviroc (UK-427857), a potent, orally bioavailable, and selective small-molecule inhibitor of chemokine receptor CCR5 with broad-spectrum anti-human immunodeficiency virus type 1 activity. Antimicrob Agents Chemother. 2005;49 (11):4721-4732.

84. Woollard SM, Kanmogne GD. Maraviroc: a review of its use in HIV infection and beyond. Drug Des Devel Ther. 2015;9:5447-5468.

85. Jayasuriya H, Herath KB, Ondeyka JG, et al. Isolation and structure of antagonists of chemokine receptor (CCR5). J Nat Prod. 2004;67 (6):1036-1038.

86. Zhang X, Haney KM, Richardson AC, et al. Anibamine, a natural product CCR5 antagonist, as a novel lead for the development of anti-prostate cancer agents. Bioorg Med Chem Lett. 2010;20 (15):4627-4630.

87. Xu GG, Zaidi SA, Zhang F, et al. Exploration on natural product anibamine side chain modification toward development of novel CCR5 antagonists and potential anti-prostate cancer agents. Bioorg Med Chem Lett. 2015;25(17):3721-3725.

88. Zhang F, Arnatt CK, Haney KM, et al. Structure activity relationship studies of natural product chemokine receptor CCR5 antagonist anibamine toward the development of novel anti prostate cancer agents. Eur J Med Chem. 2012;55:395-408.

89. Khan GJ, Rizwan M, Abbas M, et al. Pharmacological effects and potential therapeutic targets of DT-13. Biomed Pharmacother. 2018;97:255-263.
90. Lin SS, Fan W, Sun L, et al. The saponin DT-13 inhibits gastric cancer cell migration through down-regulation of CCR5-CCL5 axis. Chin J Nat Med. 2014;12(11):833-840.

91. Ren-Ping Z, Sen-Sen L, Yuan ST, et al. DT-13, a saponin of dwarf lilyturf tuber, exhibits anti-cancer activity by down-regulating $\mathrm{C}-\mathrm{C}$ chemokine receptor type 5 and vascular endothelial growth factor in MDA-MB-435 cells. Chin J Nat Med. 2014;12(1):24-29.

92. Li H, Sun L, Li H, et al. DT-13 synergistically enhanced vinorelbine-mediated mitotic arrest through inhibition of FOXM1-BICD2 axis in non-small-cell lung cancer cells. Cell Death Dis. 2017;8(5):e2810.

93. Yu XW, Lin S, Du HZ, et al. Synergistic combination of DT-13 and topotecan inhibits human gastric cancer via myosin IIA-induced endocytosis of EGF receptor in vitro and in vivo. Oncotarget. 2016;7(22):32990-33003.

94. Krensky AM, Ahn YT. Mechanisms of disease: regulation of RANTES (CCL5) in renal disease. Nat Clin Pract Nephrol. 2007;3 (3):164-170.

95. Fessele S, Maier H, Zischek C, Nelson PJ, Werner T. Regulatory context is a crucial part of gene function. Trends Genetics. 2002;18 (2):60-63.

96. Shannon P, Markiel A, Ozier O, et al. Cytoscape: a software environment for integrated models of biomolecular interaction networks. Genome Res. 2003;13(11):2498-2504.

\section{Publish your work in this journal}

OncoTargets and Therapy is an international, peer-reviewed, open access journal focusing on the pathological basis of all cancers, potential targets for therapy and treatment protocols employed to improve the management of cancer patients. The journal also focuses on the impact of management programs and new therapeutic agents and protocols on patient perspectives such as quality of life, adherence and satisfaction. The manuscript management system is completely online and includes a very quick and fair peer-review system, which is all easy to use. Visit http://www.dovepress.com/ testimonials.php to read real quotes from published authors. 